Open Peer Review on Qeios

\title{
Regenerative Surgery with Adipose Stem Cells and 3D Bioprinted Scaffolds for Cleft Palate Repair
}

\author{
Adriana Cordova ${ }^{1}$, Daniele Matta $^{1}$, Francesca Toia $^{1}$, Anna Barbara Di Stefano ${ }^{1}$ \\ 1 University of Palermo \\ Funding: The author(s) received no specific funding for this work. \\ Potential competing interests: The author(s) declared that no potential competing interests exist.
}

\begin{abstract}
Cleft lip and palate (CL/P) is the most prevalent craniofacial birth defect in humans. To date, none of the known surgical techniques and proposed therapeutic approaches is able to guarantee a definitive correction of the hard palate defect in a single surgical step. So, patients often undergo various surgical interventions and autologous bone grafts with additional scarring and further complications. The aim of regenerative medicine is to avoid the numerous surgical approaches, devising a bioengineered tissue that can be used for reconstructing the loss of substance in a quick and less invasive way. For this reason, we think that adipose tissue can be the optimal "donor tissue" and an an excellent source of adipose stem cells due to its already known regenerative properties. In our study, we want to investigate "in vitro" regenerative capacity of stem cells from adipose tissue in association with 3D bio-printed scaffolds of natural origin. The future objective will be to "package" a custom-made "negative mold" of the original hard palate/maxilla defect; this would allow us to quickly and less-invasively repair the defect, guaranteeing an excellent restitutio ad integrum and relieving the patient from the numerous complications related to multi-step surgical repair.
\end{abstract}

$\mathrm{CL} / \mathrm{P}$ has an approximate estimated incidence in Europe of about 1 case per 700 live births and it is the result of a faulty embryogenetic process $(1,2)$. Despite the great scientific interest in determining the "correct" pathology treatment strategy, great disagreement continues to exist in the scientific community concerning the "best surgery sequence" and the "correct reconstructive surgery timing". Moreover, despite the various surgical techniques proposed, the main problem continues to be hard palate/alveolar reconstruction(3,4). Lacking in the bone layer causes continuous instability in the child's palate/maxilla with cranio-facial growth impairment, feeding difficulties, chewing, swallowing and breathing and speech problems. Gold standard procedure remains the bone graft from iliac crest, taken by traditional approach or by minimally invasive approach(5). Mucous and muscular layers can be usually reconstructed through the use of local flaps. In this panorama, tissue-engineering and our study aims to re-create, in artificial conditions, tissues that are similar as possible to biological ones. This would allow to correct the bone layer defect without sacrificing healthy patient's tissues. We believe that an important role could be played by 
the S-ASCs (spheroids of adipose stem cells); In recent literature, it has been demonstrated that a population of spheroid originating from ASC in suspension possesses in-vitro a high potential for multilineage differentiation(6). We have characterized the S-ASCs demonstrating that these spheroids possess an increased early differentiation potential in all mesenchymal lineages and compared to adherent ASCs and in vivo model, they displayed enhanced properties in bone regeneration (7). We also demonstrated that the SASCs exhibit an microRNA and mRNA profile of highly undifferentiated cells, confirming that they represent a distinct upstream population of stem cells in adipose tissue(8). The preservation of the 3D structure of the cells, from extraction to administration, plays a decisive role to ensure optimal conditions for cell viability and maintenance of stemness potential(9). So, the development of 3D cell-laden hydrogels has the function to mimic the peculiar scenario of a native tissue allowing the cells to differentiate properly and survive in the new site of implant. Our study focuses on biomaterials that can be 3D bio-printed in 3D and can maintain their structural characteristics in the phases of growth and cellular organization, degrading proportionally to the patient's bone growth. We are evaluating the biocompatibility and regenerative abilities of SASCs in new vegetal-origin hydrogels, such as the Xyloglucan (XG). XG is a very common polysaccharide present in the cell walls of higher plants as a crosslinker of adjacent cellulose microfibrils and in seeds as a preservative compound. We use it to create an artificial niche by incorporating the spheroids into an injectable in situ gelling solution of partially degalactosilated XG and an ad hoc formulated culture medium for the preservation of the spheroidal characteristics of stem cells. Main limitation of XD, however, is that it cannot be 3D bio-printed, so the next step of our research is to associate XD with other materials that can maintain a three-dimensional conformation and guiding/supporting the osteoblastic differentiation of our SASCs and to study the results obtained with the various materials in order to identify the most suitable for the construction of a biointegrable support for the reconstruction of bone deficit in the cleft palate.

\section{RERENCES:}

1. Massenburg BB, Jenny HE, Saluja S, Meara JG, Shrime MG, Alonso N. Barriers to cleft lip and palate repair around the world. J Craniofac Surg. 2016;27(7):1741-5.

2. Candotto V, Oberti L, Gabrione F, Greco G, Rossi D, Romano M, et al. Current concepts on cleft lip and palate etiology. J Biol Regul Homeost Agents. 2019;33(3 Suppl. 1):145-151. DENTAL SUPPLEMENT.

3. Jia YL, Fu MK, Ma L. Long-term outcome of secondary alveolar bone grafting in patients with various types of cleft. Br J Oral Maxillofac Surg. 2006 Aug;44(4):308-12.

4. Cohen M, Polley JW, Figueroa AA. Secondary (intermediate) alveolar bone grafting. Clin Plast Surg. 1993 Oct;20(4):691-705.

5. Boyne PJ, Sands NR. Secondary bone grafting of residual alveolar and palatal clefts. J Oral Surg. 1972 Feb;30(2):87-92.

6. Jankowski M, Dompe C, Sibiak R, Wąsiatycz G, Mozdziak P, Jaśkowski JM, et al. In Vitro Cultures of 
Adipose-Derived Stem Cells: An Overview of Methods, Molecular Analyses, and Clinical Applications. Cells. 2020 Jul;9(8).

7. $A B$ DS, AA LB, A G, T A, P M. Identification and Expansion of Adipose Stem Cells with Enhanced Bone Regeneration Properties. J Regen Med. 2016;05(01).

8. Di Stefano AB, Grisafi F, Castiglia M, Perez A, Montesano L, Gulino A, et al. Spheroids from adiposederived stem cells exhibit an miRNA profile of highly undifferentiated cells. J Cell Physiol. 2018 Nov;233(11):8778-89.

9. Toia F, Di Stefano AB, Muscolino E, Sabatino MA, Giacomazza D, Moschella F, et al. In-situ gelling xyloglucan formulations as 3D artificial niche for adipose stem cell spheroids. Int J Biol Macromol. 2020 Dec;165(Pt B):2886-99. 